\title{
Measuring Packing Fraction Distribution in Cake Filtered under Constant Pressure and Quantitative Estimation from Measured Filtrate Volume
}

\author{
Takamasa MORI, Hyo-Jung KIM, Kenjiro ATO and JunIchiro TSUBAKI \\ Department of Molecular Design and Engineering, Nagoya University, Furo-cho, Chikusa-ku, Nagoya-shi 464-8603

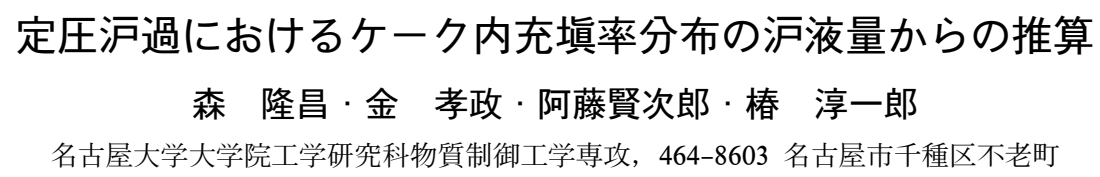

\begin{abstract}
The particle-packing structure of alumina slurry was evaluated by constant pressure filtration. The cake forming behavior were classified into four patterns by plotting the resistance of the formed cake as a function of the particle volume in the cake; the plots were (a) a straight line, (b) a convex curve, (c) a concave curve, and (d) the combination of a concave curve and a flat line. A method of a estimation of the distribution of packing fraction in the formed cake was proposed. The distribution of packing fraction in the formed cake could be calculated unless the average packing fraction of the cake increased as the filtration proceeded ((c) concave curve). It was found that the distribution of packing fraction estimated by the proposed method was in good accordance with the experimental value obtained by $\gamma$-ray transmission measurement.
\end{abstract}

[Received December 6, 2005; Accepted February 16, 2006]

Key-words : Constant pressure filtration, Packing fraction of cake, Cake forming behavior, Degree of particle dispersion or flocculation, Particle interaction

\section{Introduction}

It is important to accurately evaluate slurry properties for the optimal design of final products in wet shaping processes. ${ }^{1-3)}$ In previous studies, ${ }^{3)-5)}$ the properties of a product, such as density, can be estimated not on the basis of apparent viscosity, $\left.{ }^{6}, 7\right)$ which is one of the conventional parameters in ceramics industry, but on the particle packing ability of the slurry. ${ }^{3)}$

From this viewpoint, we proposed hydrostatic pressure measurement and analyzed particle settling and depositing behavior in detail. ${ }^{8}{ }^{89}$ (9) Furthermore, the apparent weight flux ratio was introduced for the estimation of the final packing fraction of sediment in a gravitational sedimentation test. ${ }^{10), 11 \text { ) }}$

In addition, we also reported the dependency of pressure on the packing ability of slurries by constant pressure filtration. In our previous reports, ${ }^{4,5)}$ we proposed a new analysis method of constant pressure filtration in which we focused not on the filtrate but on the particle volume in a formed cake. We reported that the average packing fraction of a formed cake can be evaluated more easily using this method. It was also shown that the filtration behaviors and the mechanisms of cake formation were classified into four patterns. ${ }^{4)}$

The objective of this study was to estimate the distribution of packing fraction quantitatively in a formed cake from the time change of filtrate and to compare the actual measurement value by the $\gamma$-ray transmission method.

\section{Experimental procedures}

2.1 Materials and slurry preparation

Slurries were prepared from $\alpha-\mathrm{Al}_{2} \mathrm{O}_{3}$ powder (AES-11E with an average particle size of $0.48 \mu \mathrm{m}$, Sumitomo Chemical) and distilled water by ball milling for $1 \mathrm{~h}$. Polyacrylic ammonium (molecular weight is $6000-10000$, Toa Gosei) was used as the dispersant. To prepare the slurries with different filtration behaviors the content in each slurry was determined on the basis of the results of a previous report. ${ }^{4)}$ The preparation
Table 1. Experimental Conditions

\begin{tabular}{cccc}
\hline Slurry & $\begin{array}{c}\text { Solid con. } \\
{[\mathrm{vol} \%]}\end{array}$ & $\begin{array}{c}\text { Adsorbed amount } \\
{\left[\mathrm{mg} \cdot\left(\mathrm{g}-\mathrm{Al}_{2} \mathrm{O}_{3}\right)^{-1}\right]}\end{array}$ & $\begin{array}{c}\text { Filtration pressure } \\
{[\mathrm{kPa}]}\end{array}$ \\
\hline 1 & 35 & 1.8 & 200 \\
2 & 35 & 2.2 & 200 \\
3 & 35 & 1.1 & 200 \\
4 & 10 & 2.2 & 100 \\
\hline
\end{tabular}

conditions are summarized in Table $\mathbf{1}$ in detail. The slurries were degassed for 10 min by vacuum treatment and then subjected to constant pressure filtration.

\subsection{Constant pressure filtration}

The prepared slurry was poured into an acrylic cylinder with an inner diameter of $35 \mathrm{~mm}$ and then pressurized at specified pressures using air. The filtration pressure was $200 \mathrm{kPa}$ for slurries 1, 2 and 3, and $100 \mathrm{kPa}$ for slurry $\left.4 .^{4}\right)$ The initial slurry volume was $90 \mathrm{ml}$ to obtain a cake with a measurable height. The time change of the filtrate was measured using an electric balance and recorded on a computer. After filtration cake thickness was measured at five different points of the cake and the average was used for the analysis.

\subsection{Measurement of packing fraction distribution}

The distribution of packing fraction in the formed cake was measured using a $\gamma$-ray densitometer (GD-5000AV, Earthnix Co.). To neglect the error caused by the drying of the cake, density was measured from the point $2 \mathrm{~mm}$ below the top surface of the cake at intervals of $5 \mathrm{~mm}$. In this method the cake density, $\rho$ can be calculated from the intensity of transmitted $\gamma$-ray, $I$, by Lambert-Beer's law as described by Eq. (1).

$$
I=I_{0} \exp (-a \rho)
$$

Here, $I_{0}$ is the intensity of incident $\gamma$-ray and $\alpha$ is the experimental coefficient determined by a calibration test using water and a slurry with a known concentration. 


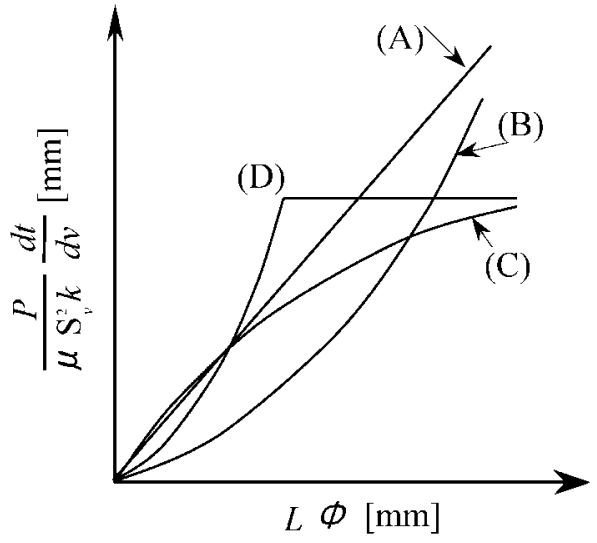

Fig. 1. Schematic illustration of filtration pattern.
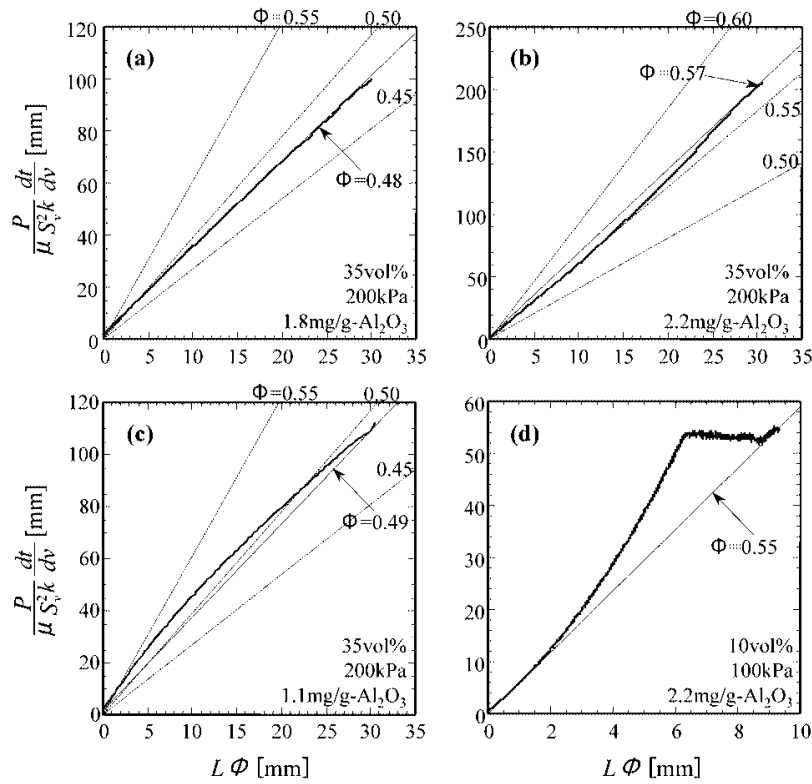

Fig. 2. Filtration patterns obtained from prepared slurries. (a) Slurry 1 (pattern A), (b) slurry 2 (pattern B), (c) slurry 3 (pattern C) and (d) slurry 4 (pattern D)

\section{Results and discussion}

\subsection{Filtration patterns}

Results of constant pressure filtration were analyzed using Eq. $(2)^{4), 5)}$ in which the variable was not the conventional filtrate $v$ but the particle volume in the formed cake $L \Phi$.

$$
\frac{P}{\mu S_{v}{ }^{2}} \frac{\mathrm{d} t}{\mathrm{~d} v}=\frac{\Phi}{(1-\Phi)^{3}}\left(L \Phi+R_{\mathrm{m}}{ }^{\prime}\right)
$$

The plot on the left side of Eq. (2) versus $L \Phi$ is called the filtration pattern. As mentioned in a previous report, ${ }^{4)}$ the filtration patterns are classified into four patterns; pattern A (an upward-sloping straight line), pattern B (a concave curve), pattern C (a convex curve), and pattern D (the combination of a concave curve and a flat line). Figure 1 shows the schematic diagram of typical filtration patterns.

Figure 2 shows the filtration patterns of prepared slurries. Slurry 1 showed pattern A (Fig. 2 (a)), slurry 2 pattern B (Fig. 2 (b)), slurry 3 pattern C (Fig. 2(c)) and slurry 4 pattern D (Fig. 2(d)).

The mechanisms of cake formation corresponding to each

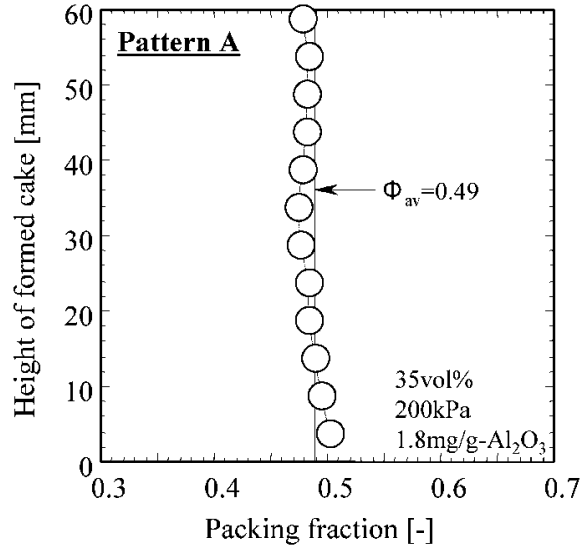

Fig. 3. Packing fraction distribution measured using $\gamma$-ray transmission densitometer for filtration pattern A.

filtration pattern have already been mentioned in elsewhere;4) thus the distribution of packing fraction in the formed cake was tried estimating from the time change of filtrate in this study. In addition, we compared the calculated values with the actual measurement values obtained by the $\gamma$-ray transmission method.

\subsection{Distribution of packing fraction in formed cake}

3.2.1 Filtration pattern $A$

For filtration pattern $\mathrm{A}$, the average packing fraction of the formed cake should be constant during filtration. ${ }^{4)}$ Figure 3 shows the distribution of packing fraction measured by the $\gamma$-ray transmission method. From this result, it was shown that the local packing fraction of the formed cake was almost constant and consistent with the average packing fraction for filtration pattern A.

\subsubsection{Filtration pattern $B$}

For filtration pattern $B$, the average packing fraction of the formed cake increased as filtration proceeded. The local solid compressive pressure at the same distance from the bottom increased with cake height; therefore, the lower layer of the formed cake was compressed by the increased compressive pressure, the average packing fraction of the cake increased as the filtration proceeded. ${ }^{4), 12)}$ However, we could not identify where in the cake and how the compression of the cake occurred.

\subsubsection{Filtration pattern $\mathrm{C}$}

For filtration pattern $\mathrm{C}$, the average packing fraction of the formed cake decreased as filtration proceeded. The total pressure drop of the cake is equal to the filtration pressure and is constant under constant pressure filtration. Therefore, the pressure gradient of cake decreased as cake height increased. If the packing fraction of the upper cake decreased with decreasing pressure gradient, the average packing fraction decreased, resulting in pattern C. ${ }^{4), 13)}$ Supposing the local packing fraction of the formed cake does not change by further compression, the distribution of packing fraction in the final cake can be calculated from the time change of filtrate as mentioned below.

Taking the differential of Eq. (2) for $\Phi$ and $v$, we obtain the following equation.

$$
\begin{aligned}
\Delta\left\{\frac{P}{\mu S_{v}^{2}} \frac{\mathrm{d} t}{\mathrm{~d} v}\right\}= & \frac{\Phi^{2}}{(1-\Phi)^{3}} \frac{\phi}{\Phi-\phi}\left\{\left(\frac{2}{\Phi}+\frac{3}{1-\Phi}\right.\right. \\
& \left.\left.-\frac{1}{\Phi-\phi}\right\} v \Delta \Phi+\Delta v\right\}
\end{aligned}
$$



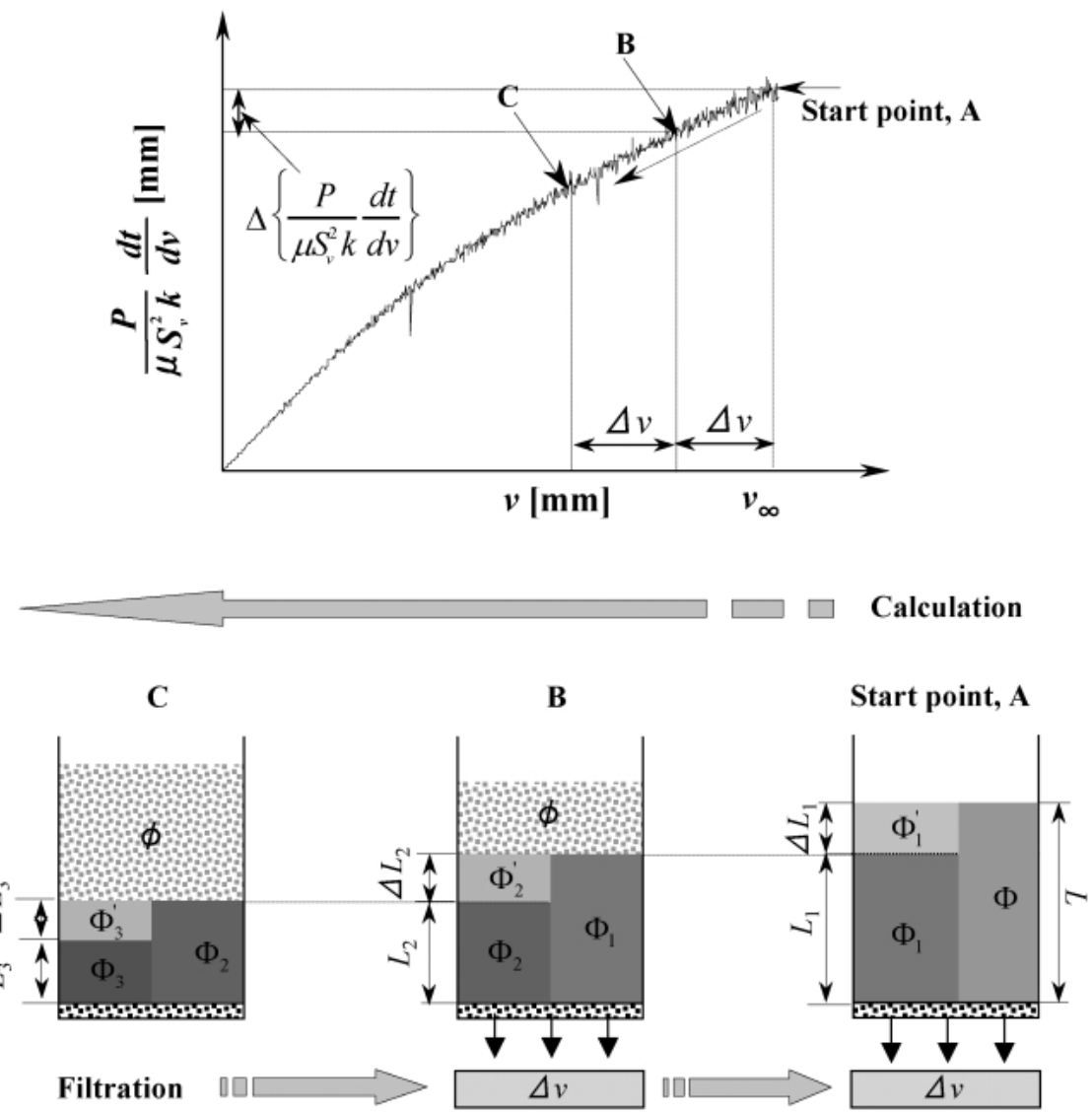

Fig. 4. Calculation model of packing fraction distribution for filtration pattern C.

We can determine the value of the left side of Eq. (3), $\Delta\{(P /$ $\left.\left.\mu S_{v}{ }^{2}\right)(\mathrm{d} t / \mathrm{d} v)\right\}$, corresponding to the given change in the filtrate, $\Delta v$, as shown in Fig. 4. Furthermore, we can calculate the average packing fraction of the final cake by the following mass balance equation because the final thickness of the formed cake was measured after filtration.

$$
L \Phi=\phi(v+L)
$$

Therefore, applying Eq. (3) to points A and B in Fig. 4, we can determine the difference in the average packing fraction of the cake between points A and B. Here, the packing fraction of the formed cake at point B, $\Phi_{1}$, does not change according to the assumption; $\Phi_{1}$ can be calculated using the following equation.

$$
\Delta \Phi=\Phi-\Phi_{1}
$$

The cake thickness $L$ also changes with the change in filtrate $\Delta v$. The change in cake thickness $\Delta L$ corresponding to $\Delta v$ can be determined using the following equation derived from the differential of Eq. (4).

$$
\Delta L=\frac{-\phi v}{(\Phi-\phi)^{2}} \Delta \Phi+\frac{\varphi}{(\Phi-\phi)} \Delta v
$$

Substituting $\Delta \Phi$ determined using Eq. (3) into this equation, the change in cake thickness between points $\mathrm{A}$ and $\mathrm{B}, \Delta L_{1}$, can be obtained. Cake thickness at point $\mathrm{B}, L_{1}$, can be determined by the following equation.

$$
\Delta L_{1}=L-L_{1}
$$

In addition, the local packing fraction of a newly formed cake after point $\mathrm{B}, \Phi_{1}$, can be determined by the material balance

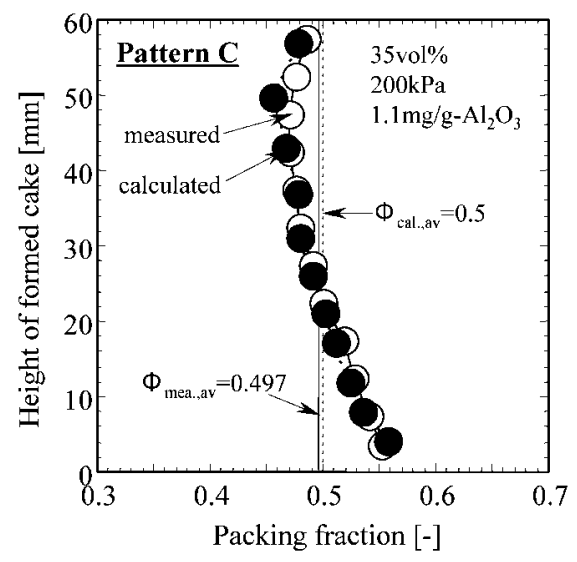

Fig. 5. Comparison of packing fraction distribution between calculated and experimental vales for filtration pattern $\mathrm{C}$.

of particles in the final cake as described by the following equation.

$$
\Phi L=\Phi_{1} L_{1}+\Phi_{1}{ }^{\prime} \Delta L_{1}
$$

The distribution of packing fraction of the formed cake at point $\mathrm{B}$ can be determined by repeating the above calculation for points B and C. Furthermore, the distribution of packing fraction in the final cake can be determined completely by repeating this calculation procedure until the starting point in Fig. 4.

Figure 5 shows the distribution of packing fraction for slur- 


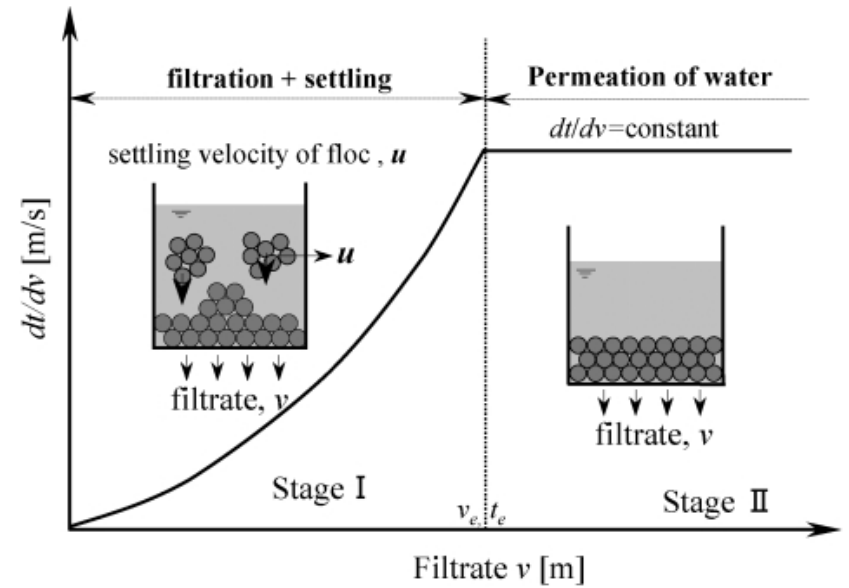

Fig. 6. Schematic illustration of cake formation for filtration pattern D.

ry 3 (Fig. 2(c)). In this figure the open marks show the measurement values obtained by the $\gamma$-ray transmission method and solid marks show the values calculated by the above procedure. The measured and calculated average packing fractions were both 0.5 , and the calculated local packing fractions were also consistent with the measured values. From this result it was shown that the local packing fraction of the formed cake decreased with an increase in cake thickness for the slurry with filtration pattern $C$.

\subsubsection{Filtration pattern D}

Figure 6 shows the schematic illustration of the cake formation mechanism for the slurry with filtration pattern D. For the slurry with filtration pattern $\mathrm{D}$, particle settling velocity cannot be neglected; therefore, most of the particles formed a cake in the middle of filtration by settling (stage I in Fig. 6) and then the liquid left above the cake passed through the cake (stage II in Fig. 6). ${ }^{4), 14)}$ In this case we should consider the effect of particle settling on the calculation of particle volume in the cake, $L \Phi$, and modify Eq. (4). Supposing that the particle settling velocity is $u$, the material balance is,

$$
\mathrm{d} L=\phi(\mathrm{d} v+\mathrm{d} L)+\phi u \mathrm{~d} t)
$$

and the integral form of Eq. (9) is

$$
L \Phi=\frac{\phi \Phi}{(\Phi-\phi)}(v+u t) \text {. }
$$

The time at which the all particles finished settling is $t_{\mathrm{e}}$, the filtrate at that point is $v_{\mathrm{e}}$, and the initial slurry height is $H_{0}$; therefore, the material balance is

$$
L \Phi=\frac{\phi \Phi}{\Phi-\phi}\left(v_{\mathrm{e}}+u t_{\mathrm{e}}\right)=\phi H_{0}
$$

Finally, the particle settling velocity $u$ is expressed by the following equation.

$$
u=\frac{1}{t_{\mathrm{e}}}\left(\frac{\Phi-\phi}{\Phi} H_{0}-v_{\mathrm{e}}\right)
$$

Substituting $u$ determined by Eq. (12) into Eq. (10), we can calculate particle volume in the formed cake take into consideration particle settling during filtration.

Figure 7 shows the revised filtration result for slurry 4. In this case, the particle settling velocity determined using Eq. (12), $u$, was $7.34 \times 10^{-7} \mathrm{~m} \cdot \mathrm{s}^{-1}$. From this result the revised filtration pattern was almost straight line, suggesting that the

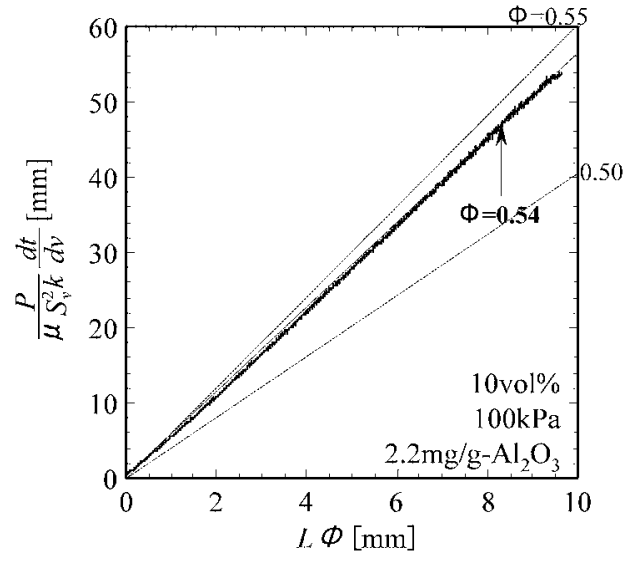

Fig. 7. Filtration pattern of slurry 4 as function of revised particle volume in formed cake.

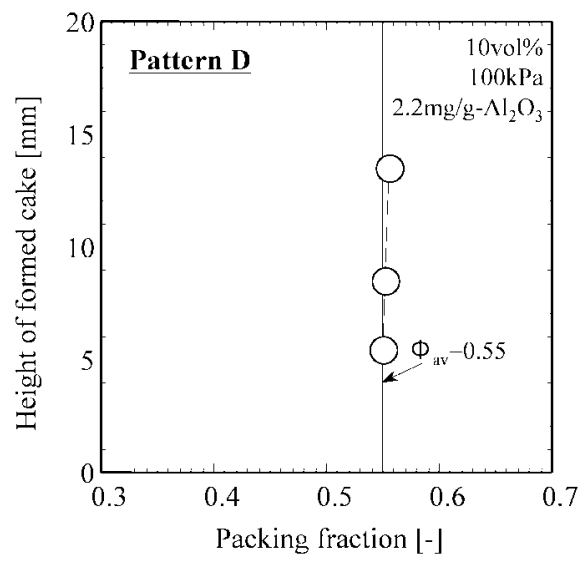

Fig. 8. Packing fraction distribution measured using $\gamma$-ray transmission densitometer for filtration pattern D.

local packing fraction of the formed cake should be constant in accordance with the same reason as that of the filtration pattern A.

Figure 8 shows the distribution of packing fraction measured by the $\gamma$-ray transmission method. From this result, it was also shown that the local packing fraction of the formed cake was almost constant and consistent with the average packing fraction for filtration pattern $\mathrm{D}$.

\section{Conclusion}

The distributions of packing fraction in the formed cake were estimated from the time change of filtrate for three typical filtration patterns; pattern A (an upward-sloping straight line), pattern $\mathrm{C}$ (a convex curve) and pattern D (a combination of a concave curve and a flat line). By comparing with the actual measurement value obtained by the $\gamma$-ray transmission method, it was shown that the calculated local packing fractions were consistent with the measured values for these three filtration patterns.

\section{Nomenclature}

$I \quad$ :intensity of detected $\gamma$-rays

$I_{0}$ : intensity of incident $\gamma$-rays

$a$ : experimental constant

$\rho$ : density of slurry or cake

$$
\begin{aligned}
& {\left[\mathrm{s}^{-1}\right]} \\
& {\left[\mathrm{s}^{-1}\right]} \\
& {\left[\mathrm{kg}{ }^{-1} \cdot \mathrm{m}^{3}\right]} \\
& {\left[\mathrm{kg} \cdot \mathrm{m}^{-3}\right]}
\end{aligned}
$$


$C_{0}$ : nonadsorbed dispersant concentration at $35 \mathrm{vol} \%$

$\begin{array}{lll}k & : \text { Kozeny constant } & {\left[\mathrm{kg} \cdot \mathrm{m}^{-3}\right]} \\ L & : \text { cake thickness } & {[-]} \\ M_{\phi}: \text { disperant dosage of } & {[\mathrm{m}]}\end{array}$

$M_{\phi}$ : dispersant dosage of slurry with concentration $\phi$

$M_{0}$ : dispersant dosage of slurry $(\phi=0.35)$

$\left[\mathrm{mg} / \mathrm{g} \mathrm{Al}_{2} \mathrm{O}_{3}\right]$

$P$ :filtration pressure

$\left[\mathrm{mg} / \mathrm{g} \mathrm{Al}_{2} \mathrm{O}_{3}\right]$

$R_{\mathrm{m}}:$ medium resistance

$R_{\mathrm{m}}{ }^{\prime}$ : coefficient defined by Eq. (3)

$[\mathrm{Pa}]$

$\left[\mathrm{m}^{-1}\right]$

$[\mathrm{m}]$

$\left[\mathrm{m}^{-1}\right]$

$S_{v}$ : specific surface area of particle

$[\mathrm{s}]$

$t$ : filtration time

$v$ :filtrate volume per unit area

$[\mathrm{m}]$

$\phi \quad$ : solid concentration of slurry

$[-]$

$[-]$

$\Phi$ : packing fraction of cake

$\mu$ :liquid viscosity

$[\mathrm{Pa} \cdot \mathrm{s}]$

\section{References}

1) Bergstron, L., Schilling, C. H. and Aksay, I. A., J. Am. Ceram. Soc., Vol. 75, pp. 3305-3314 (1992).

2) Shin, W. H., Shin, W. Y., Kim, S. I. and Aksay, I. A., J. Am. Ceram. Soc., Vol. 77, pp. 540-546 (1994).

3) Tsubaki, J., Kato, M., Miyazawa, M., Kuma, T. and Mori, H.,
Chem. Eng. Sci., Vol. 56, pp. 3021-3026 (2001).

4) Kim, H. J., Mori, T. and Tsubaki, J., J. Ceram. Soc. Japan, Vol. 113, pp. 761-767 (2005).

5) Tsubaki, J., Kim, H. J., Mori, T., Sugimoto, T., Mori, H. and Sasaki, N., J. Soc. Powder Technol. Japan, Vol. 40, pp. 438-443(2003).

6) Chou, K. S. and Lee, L. J., J. Am. Ceram. Soc., Vol. 72, pp. 1622-1627 (1989).

7) Tseng, W. and Wu, C. H., Ceramics International, Vol. 29, pp. 821-828 (2003).

8) Tsubaki, J., Kuno, K., Inamine, I. and Miyazawa, M., J. Soc. Powder Technol. Japan, Vol. 40, pp. 432-437 (2003).

9) Mori, T., Ito M., Sugimoto, T., Mori, H. and Tsubaki, J., J. Soc. Powder Technol. Japan, Vol. 41, pp. 522-528 (2004).

10) Mori, T., Kuno, K., Ito, M., Tsubaki, J. and Sakurai, T., $A d v$. Powder. Technol. (2006) in press.

11) Kim, H. J., Mori, T. and Tsubaki, J., J. Soc. Powder Technol. Japan, Vol. 41, pp. 24-30 (2004).

12) Shirato, M., Kato, H., Kobayashi, K. and Sakazaki, H., J. Chem. Eng. Japan, Vol. 3, pp. 98-104 (1970).

13) Buscall, R. and White, L. R., J. Chem. Soc., Faraday Trans. 1, Vol. 83, pp. 873-891 (1987).

14) Iritani, E., Mukai, Y. and Yorita, H, J. Chem. Eng. Japan, Vol. 25, pp. 742-746 (1999). 